\title{
Effect of prosthodontic planning on lateral occlusion scheme: a comparison between conventional and digital planning
}

\author{
Jaafar ABDUO ${ }^{1,2}$, Mohammed BENNAMOUN³ ${ }^{\text {, Marc TENNANT² }}$, John McGEACHIE ${ }^{2}$ \\ 1- Melbourne Dental School, Melbourne University, Melbourne, Australia. \\ 2- School of Anatomy, Physiology and Human Biology, University of Western Australia, Crawley, Australia. \\ 3- School of Computer Science and Software Engineering, University of Western Australia, Crawley, Australia.
}

Corresponding address: Jaafar Abduo - Melbourne Dental School - Melbourne University - 720 Swanston Street - Melbourne - Australia - Phone: 00613 90358998 - Fax: 0061393411599 - e-mail: jaafar_abduo@hotmail.com

Submitted: December 11, 2014 - Modification: February 6, 2015 - Accepted: February 25, 2015

\section{ABSTRACT}

\begin{abstract}
$\mathrm{R}^{\mathrm{cos}}$ ecently, digital wax-up is proposed as a tool to aid prosthodontic planning. However, there are no data about the effect of prosthodontic planning on lateral occlusion scheme. Objective: This study aims to evaluate the impact of conventional and digital prosthodontic planning on lateral occlusion scheme. Material and Methods: Dental models of 10 patients were collected. All models had Angle Class I occlusion and were undergoing prosthodontic treatment that would influence the lateral occlusion scheme. Each set of models had received both conventional wax-up and digital wax-up. In relation to the lateral occlusion scheme, the following variables were evaluated: the prevalence of the different lateral occlusion scheme, number of contacting teeth and percentage of each contacting tooth. Four excursive positions on the working side were included: $0.5,1.0,2.0$ and $3.0 \mathrm{~mm}$ from the maximal intercuspation position. Results: The lateral occlusion scheme of the two wax-up models was subjected to alterations following excursion. There was a tendency for the prevalence of canine-guided occlusion to increase and for the prevalence of group function occlusion to decrease with increasing excursion. The number of contacting teeth was decreasing with the increasing magnitude of excursion. For the $0.5 \mathrm{~mm}$ and $1.0 \mathrm{~mm}$ positions, the two wax-ups had significantly greater contacts than the pre-treatment models, while at the $2.0 \mathrm{~mm}$ and $3.0 \mathrm{~mm}$ positions, all the models were similar. For all models, canines were the most commonly contacting teeth, followed by the teeth adjacent to them. No difference was observed between the two wax-ups in relation to the number of contacting teeth. Conclusion: Although the prosthodontic planning had influenced the pattern of the lateral occlusion scheme and contacts, there was no difference between the conventional and digital prosthodontic planning.
\end{abstract}

Keywords: Diagnostic techniques. Wax. Dental occlusion. Dental models.

\section{INTRODUCTION}

The ideal lateral occlusion scheme has been a topic of debate for many years. It has been postulated that the lateral occlusion scheme impacts masticatory function, comfort and aesthetics. Several authors discussed the rationale of each lateral occlusion scheme ${ }^{19,23}$. It is common throughout prosthodontic treatment that the lateral occlusion scheme be altered by controlling morphologies, alignments and orientations of teeth.
The two commonly applied schemes are canineguided occlusion and group function occlusion. The canine-guided occlusion is a mutually protected occlusion where the vertical and horizontal overlap of the canine teeth causes disengagement of the posterior teeth in the lateral movement of the mandible ${ }^{9}$. The group function occlusion is based on multiple contacts between the maxillary and mandibular teeth in lateral movement on the working side ${ }^{9}$. It has been speculated that canine-guided occlusion protects the posterior 
teeth laterally because of the canines' strategic location, anatomy and proprioceptive properties ${ }^{19}$. On the other hand, group function occlusion might contribute to a wide distribution of occlusal forces on several teeth instead of a single tooth; thus, the occlusion can be more comfortable and functional23. However, true clinical evidence supporting either scheme is still lacking, therefore, both schemes are deemed acceptable 4,24 .

More recently, there has been a discussion about the limitations of defining each lateral occlusion scheme, as the occlusal presentation is naturally more complex ${ }^{17}$. For example, with different degrees of excursion, the lateral occlusion scheme might differ. Further, any functional occlusion is subjected to changes with time, yet without manifestation of physiological abnormalities ${ }^{2}$. It is also acknowledged that most patients are comfortable with their existing dentition and occlusion. Thus, the occlusion scheme can be considered physiological even if it does not fit into any specific category.

Fixed prosthodontic treatment is indicated to alter the tooth morphology, which can eventually alter the lateral occlusion scheme. To date, the authors are not aware of any study that evaluated the impact of fixed prosthodontic treatment on the lateral occlusal scheme. The purpose of this observational study is to evaluate the effect of two forms of fixed prosthodontic planning (conventional and digital diagnostic wax-ups) on lateral occlusion scheme. Further the frequency of each tooth contact will be quantified. The null hypotheses are that the prosthodontic planning will change the lateral occlusion scheme and the frequency of teeth contacts, and there will be a difference between the two forms of prosthodontic planning.

\section{MATERIAL AND METHODS}

Models of 10 patients who required fixed prosthodontic treatment (crowns or fixed partial dentures) were collected for this study. Figure 1 summarizes the inclusion criteria. The treatment indications were managements of heavily restored dentition, tooth wear, aesthetic problems and shortspan edentulous area. For all models, the diagnostic wax-up had an influence on the lateral occlusion scheme. The patients received the treatment at the Oral Health Centre of Western Australia and a human research ethics approval was obtained from the Human Research Ethics Committee of the University of Western Australia (RA/44/1/5079).

An irreversible hydrocolloid impression (Alginate, GC America, IL, USA) was taken for each arch and an occlusal relation record was obtained by intra-oral polyvinyl siloxane registration material (GC Exabite, GC America, IL, USA) according to the centric relation position. All the impressions were poured by type III dental stone (Buff Stone, Adelaide Moulding \& Casting Supplies, South Australia, Australia). These casts comprised the pre-treatment models. All the models were duplicated twice by reversible hydrocolloid duplicating materials (Magafeel, MKM System, Haanova, Slovakia). On one model, the conventional wax-up was executed and the other model was used for the digital wax-up (Figure 2).

\section{Conventional wax-up}

On a semi-adjustable articulator (Whip Mix, Louiseville, KY, USA), one set of duplicate models were articulated according to maximal intercuspation to represent the pre-treatment articulation. The other sets were used for the conventional wax-up and were articulated according to centric relation position with the aid of the intraoral record. Therefore, after the wax-up, there was be a coincidence between centric relation and maximal intercuspation positions ${ }^{4}$. Silicone material putty (Dental Speedex Putty, Coltene/Whaledent AG, Altstatten, Switzerland) was applied on to the buccal aspects of the posterior teeth of the mounted models. This silicone index was used for the digital articulation of the pre-treatment models.

The conventional wax-up procedure involved inlay wax addition on the external tooth surface with the aim of rectifying the defective tooth structure, obtaining natural and aesthetic tooth morphology, achieving symmetry between the two sides, and obtaining even bilateral occlusal contacts. For some patients, an increase of the vertical dimension of occlusion was necessary. All the conventional wax-ups were completed by an experienced dental technician. The dental technician was advised to

\begin{tabular}{|l|}
\hline Requirements of fixed prosthodontic treatment in the form of crowns or fixed partial dentures in at least one arch \\
\hline Diagnostic wax-up is indicated prior to the treatment \\
\hline Angle Class I occlusal relationship \\
\hline Well-distributed occlusal contacts \\
\hline Absence of temporomandibular joint disorder \\
\hline The planned prosthesis is completely supported by natural teeth \\
\hline No removable or implant prosthesis \\
\hline
\end{tabular}

Figure 1- Inclusion criteria 
produce a group function lateral occlusion scheme for the initial excursions with no steep occlusion guidance. On the completed wax-up models, silicone indices were formed on the buccal aspect of the posterior teeth.

A micro-CT scanner (SkyScan, Bruker micro $\mathrm{CT}$, Kontich, Belgium) was used to scan the pretreatment, conventional wax-up models and the associated silicone indices. The generated Digital Imaging and Communication Medicine (DICOM) images were used to construct virtual 3D Stereolithography (STL) images of the maxillary and mandibular models by a DICOM viewing program (CTvox, Bruker microCT, Kontich, Belgium). The virtual image of the conventional wax-up was used for the subsequent analysis (Figure 2B).

In order to digitally articulate the models, the image registration process by a 3D rendering software package (Geomagic Studio, Raindrop Geomagic Inc., Research Triangle Park, NC, USA) was applied. Initially, corresponding points on common surfaces between the model and the silicone indices were selected to approximate the positions of the images. This was followed by implementing the Iterative Closest Point Algorithm, which re-orients the models according to the best fit principles. The same process was repeated for the other silicone index and the opposing arch. Eventually, the silicone indices were deleted digitally, and the two models were digitally articulated.

\section{Digital wax-up}

The digital wax-up has been discussed in an earlier report ${ }^{1}$. In summary, the pre-treatment models and the associated silicone indices were scanned and converted to virtual images. The virtual pre-treatment models were articulated according to the centric relation position. This was achieved by superimposing the virtual pre-treatment models on the virtually articulated conventional wax-up models. The unaltered tissues were used for the registration. A 3D rendering software (Geomagic Studio) was used to execute the digital wax-up. The pre-treatment models were articulated by the process of Image Registration. Virtual physiological teeth (Phonares Teeth, Ivoclar Vivadent AG, Schaan, Liechtenstein) were used to alter the teeth morphologies. The virtual tooth alignment involved size alteration, rotation and translation. Each virtual tooth was fitted manually on the model with the aim of obtaining ideal teeth arrangement, emergence profile, symmetry and aesthetics. After the completion of the wax-up, the scanned model and the virtual teeth were merged to generate a single model (Figure 2C), which enhanced the computation speed of the subsequent analysis.

\section{Virtual simulation of lateral movement}

For each set of articulated models, the occlusion scheme was evaluated for each working side separately. From the maximal intercuspation position (Figure 3A), four horizontal excursive positions were considered: $0.5 \mathrm{~mm}, 1.0 \mathrm{~mm}, 2.0$ $\mathrm{mm}$ and $3.0 \mathrm{~mm}$ (Figure $3 \mathrm{~B}$ to Figure $3 \mathrm{E}$ ). The lateral movement was simulated virtually by moving the mandibular arch in the working side horizontally for each specified location. This was followed by gradually moving the mandible vertically away from the maxilla by $0.05 \mathrm{~mm}$ increments. Once all the contacts on the working side disappeared,
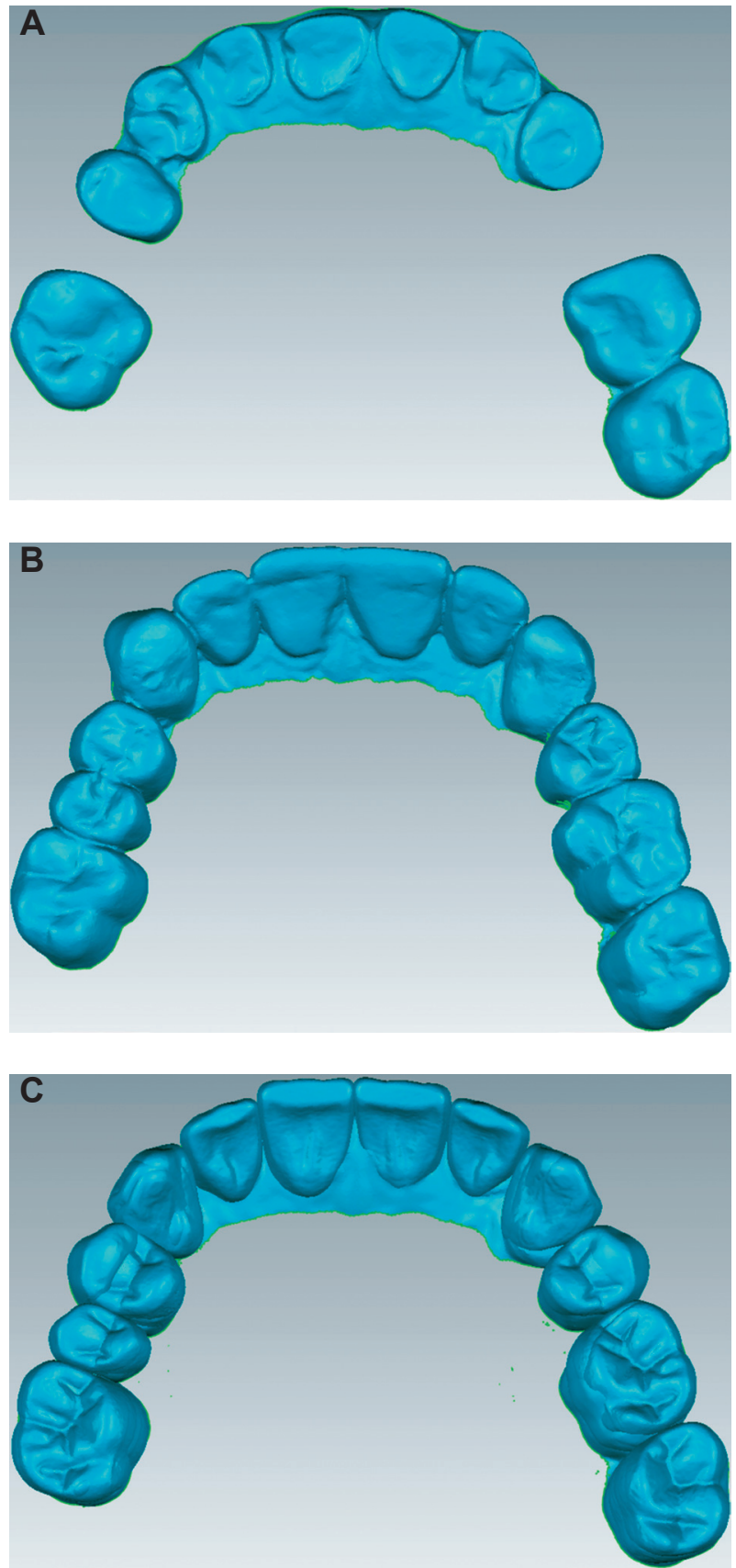

Figure 2- Example of the evaluated virtual models. (A) Pre-treatment model; (B) Conventional wax-up model; (C) Digital wax-up model 
the mandible was moved by $0.05 \mathrm{~mm}$ vertically towards the maxilla7. This process detected the existing working side contacts that dictate the lateral occlusion scheme. Due to the limitation of this process in detecting non-working side contacts, only the working side contacts were considered. Depending on the detected contacts, three occlusion schemes were considered: (1) canine-guided occlusion, (2) group function occlusion, and (3) single tooth-guided occlusion. Canine-occlusion was recorded if the lateral contact occurred on a canine tooth. The occlusion is considered group function occlusion if more than two teeth in one arch were contacting laterally. In situations where the lateral contact occurred on a single tooth other than the canine, the lateral occlusion scheme was considered to be a single tooth-guided occlusion.

\section{Analysis}

For each position, three variables were evaluated: (1) the prevalence of each lateral occlusion scheme (2) the average number of contacting teeth and (3) the percentage of each contacting tooth. All the variables were blotted in bar diagrams. For the last two variables, the maxillary and mandibular teeth contacts were distinguished. In addition, for the average number of contacting teeth, the KruskallWallis test was used to determine the presence of a statistical difference between the different positions $(p=0.05)$. When a significant difference was observed, the Mann-Whitney test was used for post-hoc analysis. Further, the difference in the number of teeth in contact between the pretreatment models and each of the two wax-up models, and between the two wax-up models was
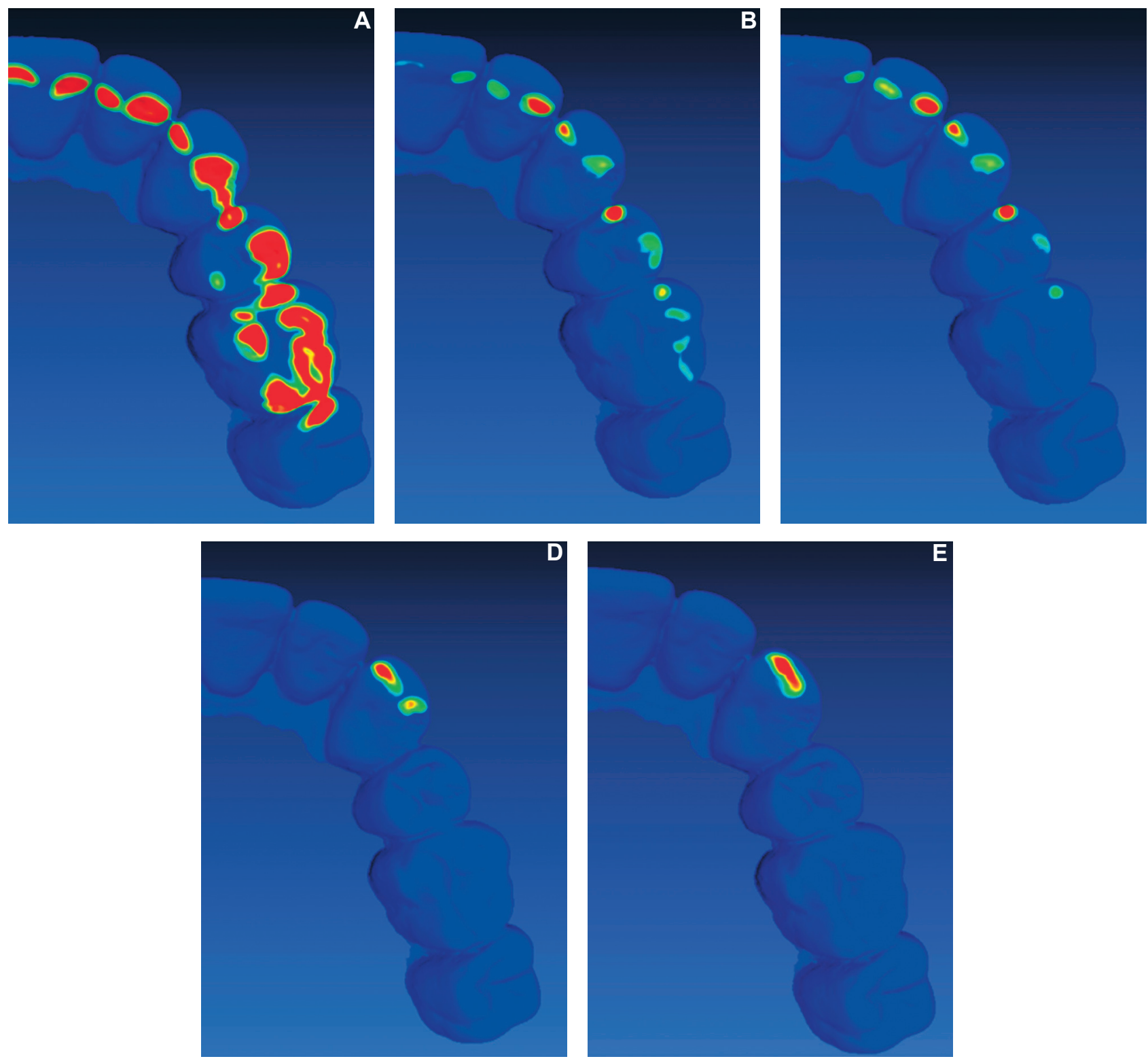

Figure 3- Example of virtual simulation of lateral movement. (A) Maximal intercuspation; (B) $0.5 \mathrm{~mm}$ excursion; (C) $1.0 \mathrm{~mm}$ excursion; (D) $2.0 \mathrm{~mm}$ excursion; (E) $3.0 \mathrm{~mm}$ excursion; red colour indicates the existing contacts 
evaluated by the Mann-Whitney test $(p=0.05)$. The same test was applied to evaluate the difference between maxillary and mandibular arches for each position.

\section{RESULTS}

\section{Prevalence of lateral occlusion scheme}

As the excursion increases, the lateral occlusion scheme changes for all the evaluated models (Figure 4). Overall, the models exhibited similar patterns of lateral occlusion alterations. For the pre-treatment models (Figure 4A), the prevalence of canine-guided occlusion had increased minimally through the excursion (from $30 \%$ to $45 \%$ ). There was a tendency for the group function occlusion to reduce with increased excursion (from $65 \%$ to $20 \%$ ). The single tooth occlusion had a tendency to increase with increased lateral excursion.

For the two wax-up models (Figure 4B and Figure $4 \mathrm{C})$, there was a consistent and gradual increase of canine-guided occlusion (from $20 \%$ to $60 \%$ for conventional wax-up, and from $0 \%$ to $65 \%$ for digital wax-up) and a reduction of group function occlusion (from $75 \%$ to $15 \%$ for conventional waxup, and from $95 \%$ to $29 \%$ for digital wax-up) with increased excursion. It appears that the single tooth occlusion had minimally increased as the excursion increased. The overall patterns for the two wax-ups were similar, except that the conventional wax-up

A

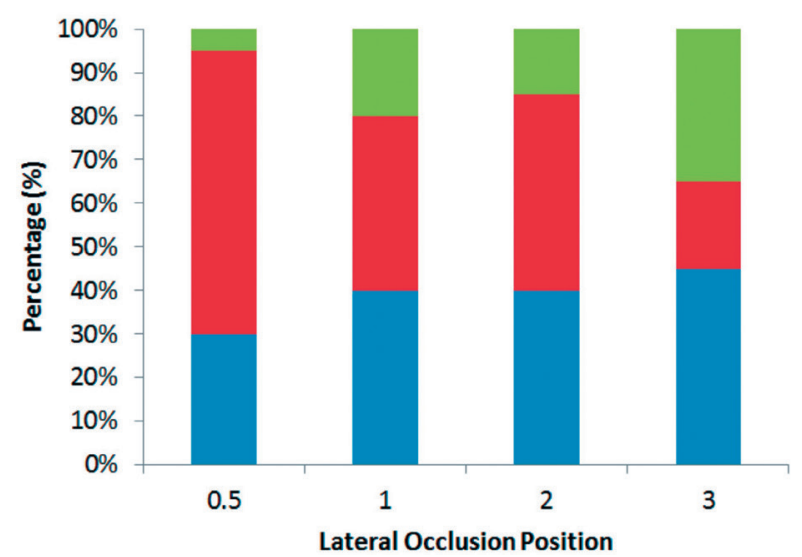

Single tooth-guided occlusion

- Group function occlusion

- Canine-guided occlusion

B

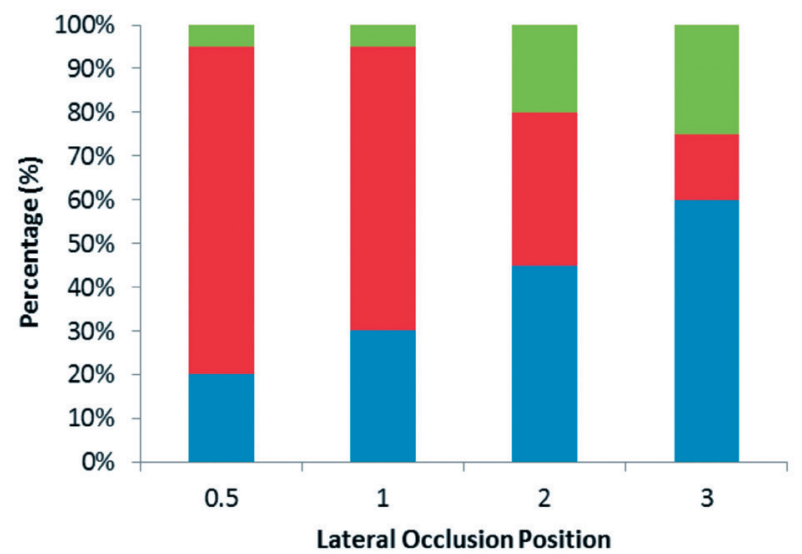

\author{
Single tooth-guided occlusion \\ - Group function occlusion \\ - Canine-guided occlusion
}

C

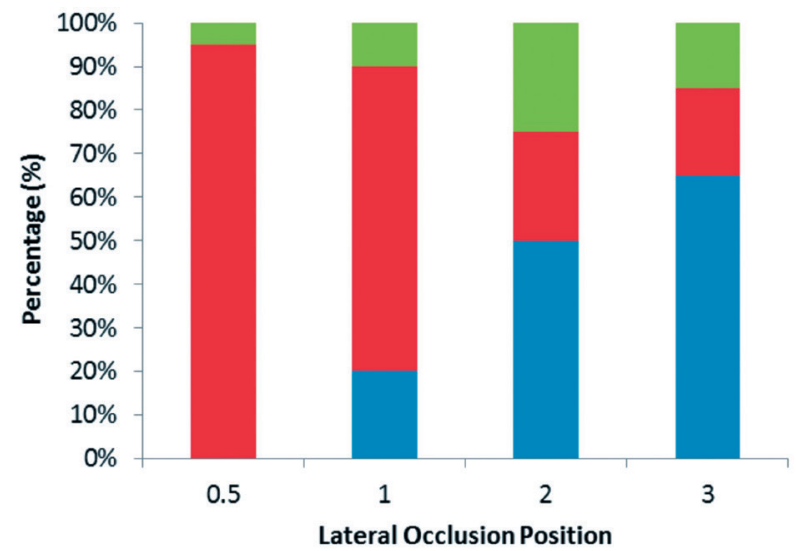

- Single tooth-guided occlusion

- Group function occlusion

- Canine-guided occlusion

Figure 4- Proportion of each lateral occlusion scheme in each excursive position. (A) Pre-treatment models; (B) Conventional wax-up models; (C) Digital wax-up models 
had a slightly more even pattern of alteration, while the digital wax-up exhibited steeper occlusion alterations.

\section{Number of contacting teeth}

Regardless of the evaluated model, there was a clear pattern of reduction of the average number of teeth in contact by increasing the degree of excursion (Figure 5). For the pre-treatment models, there was a slight reduction in the number of teeth in contact by increasing the degree of excursion, yet there was a statistical difference between all the positions, except between $1.0 \mathrm{~mm}$ and $2.0 \mathrm{~mm}$. For the two wax-ups, there was a clear nominal reduction in the number of teeth in contact by increasing the excursion. A statistically significant difference was observed between all the positions, except between 2.0 and $3.0 \mathrm{~mm}$, for the digital wax-up. Between the maxillary and mandibular arches, no statistically significant difference was observed in any position for pre-treatment and the two wax-up models.

At the $0.5 \mathrm{~mm}$ and $1.0 \mathrm{~mm}$ excursion positions, the pre-treatment models had significantly less teeth contacting than the two wax-up models.
However, at the $2.0 \mathrm{~mm}$ and $3.0 \mathrm{~mm}$ positions, there was no difference between the pre-treatment and the two wax-up models. At all the positions, there was no statistical difference between the conventional and digital wax-ups.

\section{Percentage of each contacting tooth}

For all the arches of all the models, and almost at all positions, the canine had the tendency to be the dominant contacting tooth. Further, it appears that the contacts decreased gradually from canines to the more anterior teeth and from the canines to the posterior teeth. However, there were some differences in the frequency and patterns of contacts (Figure 6).

For the pre-treatment models (Figure 6A and Figure $6 \mathrm{~B}$ ), the maxillary arch had frequent canine contacts at all the positions. The lateral incisors, premolars and the first molars had consistent contact at all the positions. In the mandible, there was a reduction in the percentage of canine contacts. Overall, there was similarity in contact frequency between the canines and premolars.

For the conventional wax-up (Figure 6C and Figure 6D), there was a general similarity between

A

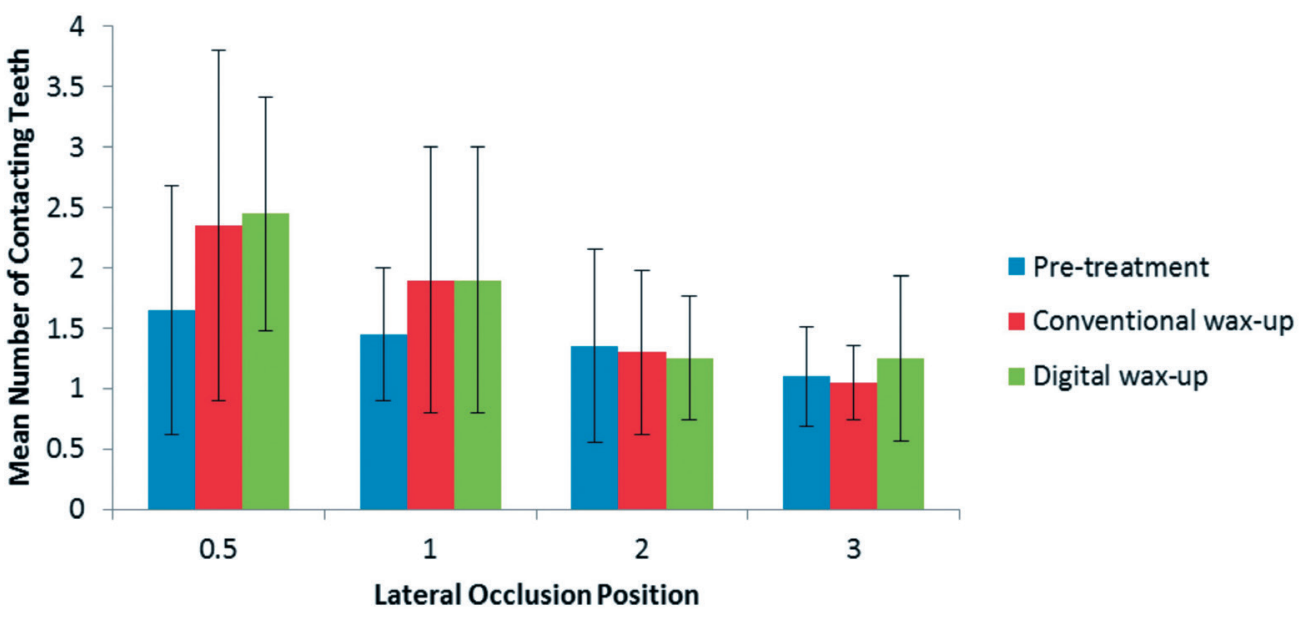

B

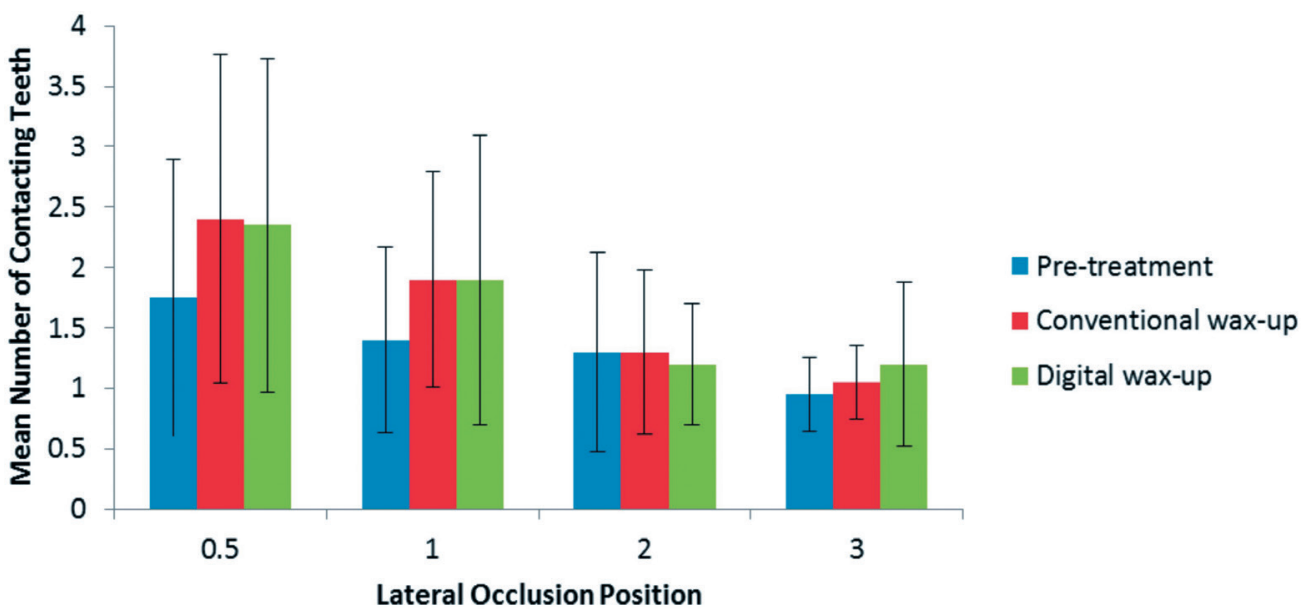

Figure 5- The mean number of contacting teeth for all the models in each excursive position. (A) Maxillary arch; (B) Mandibular arch 

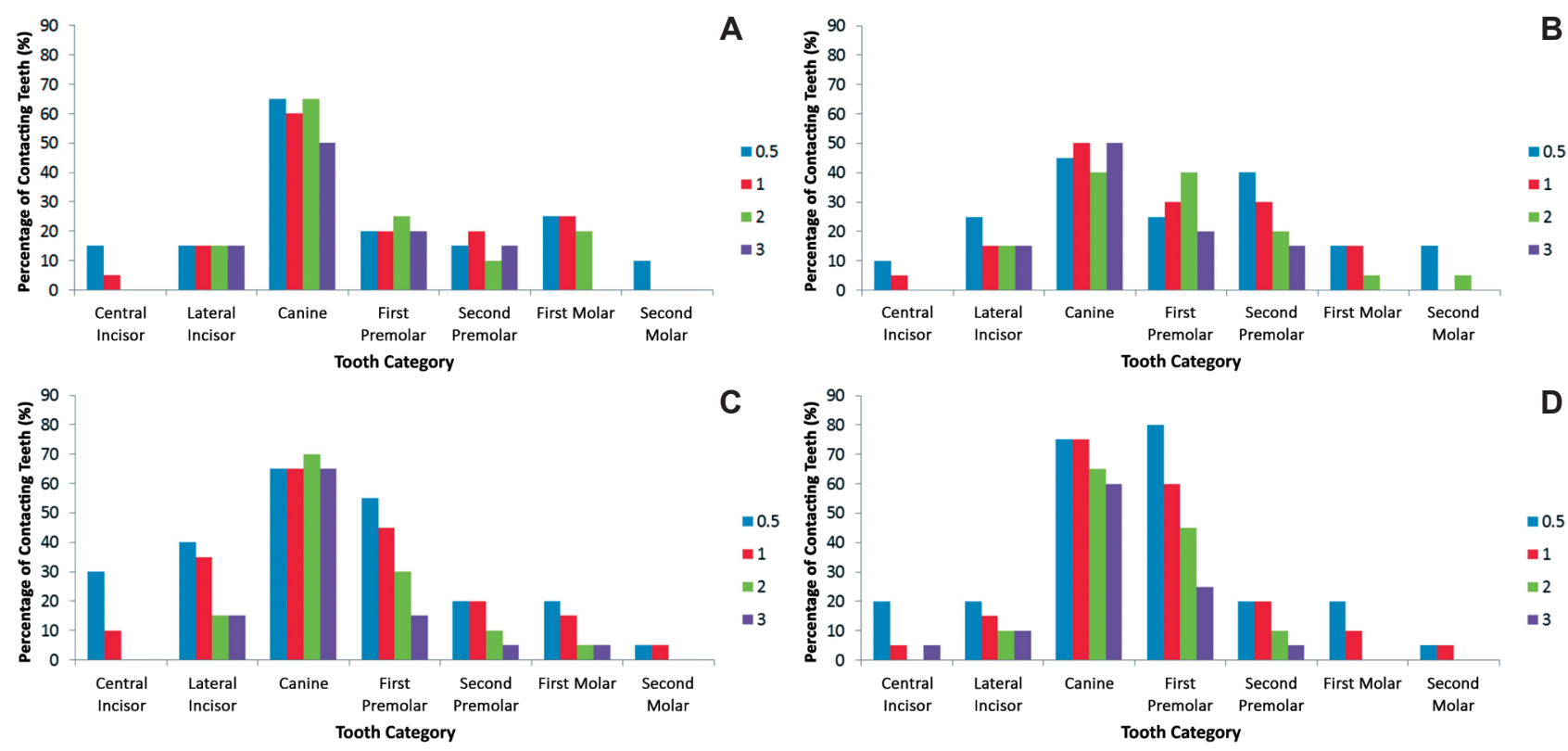

D
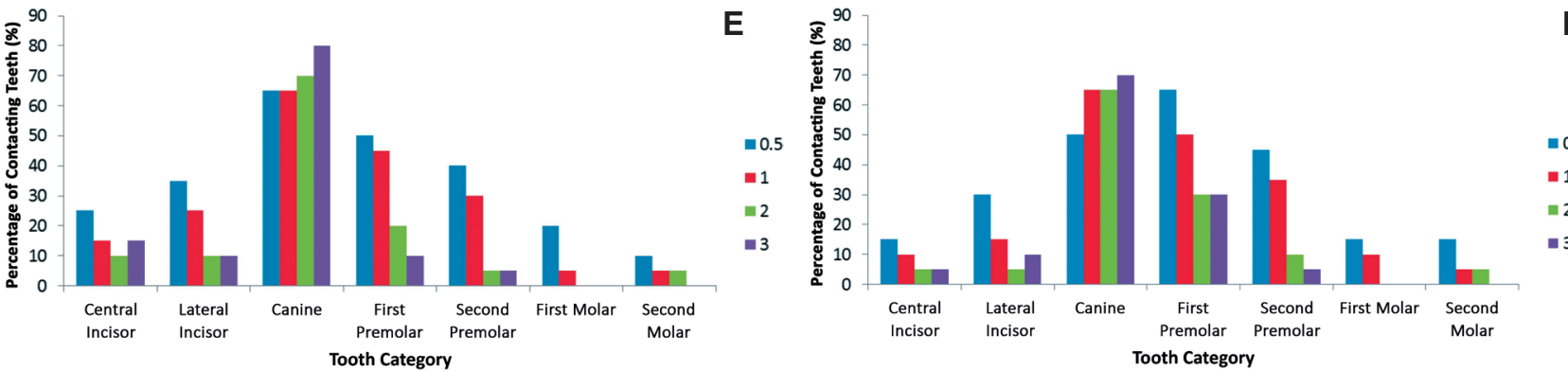

Figure 6- Percentage of the contacting teeth in each excursive position for all the models. (A) Pre-treatment maxillary arch; (B) Pre-treatment mandibular arch; (C) Conventional wax-up maxillary arch; (D) Conventional wax-up mandibular arch; (E) Digital wax-up maxillary arch; (F) Digital wax-up mandibular arch

the two arches. The canines maintained the frequency of contacts between the positions. The first premolar was the second tooth in the frequency of contacts. For all the remaining teeth, it was clear that the frequency of contacts had a tendency to decrease with increasing the excursion position. The mandibular canines were distinguished in the slight reduction of frequency with increased excursion.

In relation to the digital wax-up (Figure $6 \mathrm{E}$ and Figure 6F), the two arches showed similar patterns and frequency. The frequency of the canines contacts had increased with excursion, while the remaining teeth contact frequency had decreased significantly with excursion.

\section{DISCUSSION}

This study indicates that conventionally and digitally planned prosthodontic treatment influence the lateral occlusion in relation to the prevalence of each scheme at different positions, and the contacting teeth type and quantity. Therefore, the hypothesis that the prosthodontic planning will impact the dynamic occlusion was accepted. Such a finding supports that the lateral occlusion scheme should be carefully considered before and after the treatment.

In this study, multiple lateral locations were considered in order to provide an insight about the possible contact pattern from the immediate excursion to the maximal excursion (about $3 \mathrm{~mm})^{17}$. This was found to be more clinically relevant than only evaluating the lateral occlusion at maximal excursion ${ }^{22,27}$. Guiding contacts at the $3 \mathrm{~mm}$ position might occur primarily during parafunctional activity and bruxism in the edge-to-edge position. However, occlusal guiding during mastication and physiological movement tends to occur within 0.5 $\mathrm{mm}$ from the maximal intercuspation ${ }^{15,16}$. Therefore, the range evaluated in this study covers functional and parafunctional jaw movement.

In relation to the prevalence of each lateral occlusion scheme, it is clear that with greater excursion, the prevalence of canine-guided occlusion tends to increase. This was in accordance with the studies that evaluated the prevalence of each lateral occlusion scheme at different positions for the natural dentitions of young participants 3,28 . Yaffe and Ehlrich ${ }^{28}$ (1987) evaluated the prevalence of the different lateral occlusion schemes at 1.0, 
2.0 and $3.0 \mathrm{~mm}$ excursions. They found that the prevalence of canine-guided occlusion had increased from $16.1 \%$ to $48.6 \%$, and the group function occlusion had reduced from $83.9 \%$ to $51.5 \%$. A similar finding was observed by Al-Nimri, et al. ${ }^{3}$ (2010), when they evaluated the prevalence of the lateral occlusion scheme at 0.5 and $3 \mathrm{~mm}$ positions. They found that the prevalence of canine-guided occlusion had increased from $21.9 \%$ to $59.6 \%$, and the prevalence of group function had reduced from $45.3 \%$ to $23.9 \%$. Although the present study evaluated the lateral occlusion scheme of restored dentitions, overall, the figures supported the outcome of the earlier studies. The differences in documenting the prevalence of each occlusion scheme could be related to the different classification and recording methods of the lateral occlusion scheme ${ }^{2,17}$. In addition, it is important to emphasize that a single lateral occlusion scheme did not exist for any model for the entire excursive path. Therefore, in accordance with all the published clinical studies, true canine-guided occlusion or group function occlusion seldom exists clinically 2,4,24,27.

The dynamic nature of the lateral occlusion scheme at the different arch positions is attributed to teeth morphological factors. In the initial phase of excursion, the cusps are articulated against wider fossa surfaces ${ }^{21}$. As excursion progresses, the total contact area is reduced, which means less teeth will be in contact. This observation supports the concepts of "progressive occlusion", in which many teeth initially control the occlusion, followed primarily by the canines during the maximal excursion $^{8,10}$. This occlusion scheme appears to be more physiologically relevant than a single occlusion scheme that controls the lateral movement through the entire excursion. This complex relationship might have a protective role in tolerating lateral forces ${ }^{28}$. Physiologically, it is thought that such an arrangement is advantageous in facilitating smooth multidirectional movement of the mandibular arch, which might reduce the risk of patient discomfort ${ }^{21}$. Further, broad occlusal contact areas were found to be helpful in mitigating excessive occlusal forces on teeth ${ }^{11}$, which might contribute to the dissipation of sudden lateral forces on the teeth. In addition, the greater prevalence of group function occlusion means that greater contacts at less lateral movement can enhance the occlusal phase of chewing 25 .

The pattern of lateral occlusion scheme alteration with different excursion was observed for the pretreatment and wax-up models. However, the waxup models had more consistent gradual occlusion scheme changes. This might be due to the pretreatment dentitions suffering from morphological abnormalities, such as tooth wear or failed restorations. Subsequently, a wider contact area between worn down teeth might be evident and will maintain a greater number of contacts, even after maximal excursion ${ }^{5}$. Whereas the dentitions of the wax-up models had restored natural dental morphology and less prominent wear facets, which can produce steeper articulation and cause a consistent reduction of the total number of tooth contacts during excursion. This was evident in the initial phases of excursion $(0.5-1.0 \mathrm{~mm})$, in which the contacts quantity was significantly greater for the two wax-up models than at the later stages of excursion. In addition, it was clear that the proportion of canine-guided occlusion at later stages of excursion was greater for the two wax-ups than for the pre-treatment models. This was also likely to be related to restoring the cuspal morphology of the canines to the original canine morphology. The studies that evaluated the implication of individual age on excursion scheme confirmed that the younger the individual, the greater the prevalence of canine-guided occlusion ${ }^{2,18}$. This was attributed to the prominent dental morphology and less cuspal wear of the young dentitions $\mathbf{2}^{2,18}$. Therefore, it could be speculated that the planned prosthodontic treatment can return the dentition occlusion scheme closer to its original state. Between the two waxups, some differences were observed, but not to a significant level. Thus, it is difficult to assume that a different wax-up protocol will produce a different outcome on the final treatment. Therefore, the hypothesis that there will be a difference between the two prosthodontic planning procedures was rejected.

It is clear that at all positions, the canines had the greatest number of contacts. The significant contribution of canines was observed even after partial excursion, where group function occlusion was dominating. This reflects the importance of canines for lateral occlusion scheme development. This finding corroborates the observation by several earlier investigations ${ }^{12,13,17,28}$. Ogawa, et al. ${ }^{17}$ (1998), on natural and young dentition, found that the canines were contacting in about $70 \%$ of the excursive positions, which was similar to the outcome of our pre-treatment models in the present study. Such a finding supports the protective role of canines during the lateral occlusal movements. The dominance of canines is related to two factors: the canine's morphology and position. The canines are innately long teeth, which tend to have more prominent cuspal morphology, which controls the articulation against the opposing teeth ${ }^{19}$. As the excursion starts, the canines are in contact; however, with increased excursion the canines will play a greater role and it is more likely for the other teeth to be out of occlusion. As the working side condyle rotate and the non-working 
side condyle slides, the working side canine is positioned in the corner, where interferences control occlusal guidance ${ }^{6}$. The importance of the location in the arch explains the reason why the teeth immediately adjacent to the canine tend to have frequent occlusal contacts. Likewise, as the teeth are more distant from the canines, they are less likely to be in contact on the working side ${ }^{6,17}$. Although some investigators had found that group function was more common, for a great portion of their participants the group function occlusion was composed of canine and premolar occlusion, which reinforces the importance of the location in the $\operatorname{arch}^{12,13,26}$. The strategic importance of canines is further supported by being the most vulnerable teeth for attrition ${ }^{19,26}$. Therefore, regardless of the observed lateral occlusion scheme, it could be stated that the canines are significant teeth to control the lateral occlusion ${ }^{19}$.

Following the prosthodontic planning, it was clear that the frequency of the canine contact was increased, followed primarily by the first premolars. As stated earlier, this was attributed to restoring the original canine cuspal morphology, while the canines of pre-treatment models suffered from greater tooth wear ${ }^{5}$. Therefore, after the prosthodontic planning, a greater protective role of canines was more evident. The two wax-ups were very similar in the prevalence of the lateral occlusion scheme and the pattern of occlusal contacts frequency. However, for the digital wax-up, there appeared to be an exaggerated role of the canine teeth from the greater frequency of canine contacts. The most likely explanation is the well-defined dental morphologies that can be attained digitally by the software ${ }^{14}$. On the other hand, this means that the digital wax-up can produce a steeper lateral occlusion than the conventional wax-up, which can result in more restricted lateral movement. Although this may have clinical implications, like patient discomfort ${ }^{21}$, such assumption should be confirmed by additional studies.

Despite the lack of significant statistical difference between the two wax-up protocols applied in this study, it is important to reinforce that the digital wax-up is still in its early phase of application and requires additional investigation. For example, for the digital wax-up to be applicable clinically, the models have to be produced by computer-aided manufacturing, which will inevitably influence the accuracy of the lateral occlusal contacts. Further, the methodology of this study is limited by not considering the non-working side contacts, which could influence the observed lateral occlusion scheme. The omission of observing the non-working side contacts was necessary, as virtual modelling of the condylar movement is very imprecise ${ }^{20}$. However, as the digital wax-up is associated with steeper occlusal surfaces, the likelihood of developing non-working side contacts is higher.

\section{CONCLUSION}

Given the limitations of the present study, the following can be concluded:

The prosthodontic planning influenced the pattern of the lateral occlusion scheme. The influence of the alteration tends to be more prominent at the initial stages of excursion.

For all the models, the initial phase of excursion involved a greater number of contacting teeth and higher prevalence of group function occlusion than maximal excursion. Canine-guided occlusion tends to be more prevalent at the later stage of excursion.

Overall, the difference in the number and pattern of contacts is very minimal between the two waxups.

\section{ACKNOWLEDGEMENTS}

This research is supported by the Research Development Award from the University of Western Australia, and the Australian Prosthodontic Society. The authors acknowledge the facilities, the scientific and technical assistance of the National Imaging Facility at the Centre for Microscopy, Characterization \& Analysis, University of Western Australia; a facility funded by the University, State and Commonwealth Governments. The authors would like to thank the staff at the technical facility of the Oral Health Centre of Western Australia.

\section{REFERENCES}

1- Abduo J, Bennamoun M, Tennant M, McGeachie J. Precision of virtual prosthodontic planning for oral rehabilitation. Br J Appl Sci Technol. 2014;4(27):3915-29.

2- Abduo J, Tennant M, McGeachie J. Lateral occlusion schemes in natural and minimally restored permanent dentition: a systematic review. J Oral Rehabil. 2013;40(10):788-802.

3- Al-Nimri KS, Bataineh AB, Abo-Farha S. Functional occlusal patterns and their relationship to static occlusion. Angle Orthod. 2010;80(1):65-71.

4- Becker CM, Kaiser DA, Schwalm C. Mandibular centricity: centric relation. J Prosthet Dent. 2000;83(2):158-60.

5- Beyron HL. Occlusal changes in adult dentition. J Am Dent Assoc. $1954 ; 48(6): 674-86$.

6- D'Amico A. Functional occlusion of the natural teeth of man. J Prosthet Dent. 1961;11(5):899-915.

7- DeLong R, Heinzen M, Hodges JS, Ko CC, Douglas WH. Accuracy of a system for creating 3D computer models of dental arches. J Dent Res. 2003;82(6):438-42.

8- DiPietro GJ. A study of occlusion as related to the Frankfortmandibular plane angle. J Prosthet Dent. 1977;38(4):452-8.

9- The glossary of prosthodontic terms. J Prosthet Dent. 2005;94(1):10-92.

10- Goldstein GR. The relationship of canine-protected occlusion to a periodontal index. J Prosthet Dent. 1979;41(3):277-83.

11- Hidaka O, Iwasaki M, Saito M, Morimoto T. Influence of clenching intensity on bite force balance, occlusal contact area, and average bite pressure. J Dent Res. 1999;78(7):1336-44. 
12- Ingervall $B$. Tooth contacts on the functional and nonfunctional side in children and young adults. Arch Oral Biol. 1972;17(1):191200.

13- Ingervall B, Hähner R, Kessi S. Pattern of tooth contacts in eccentric mandibular positions in young adults. J Prosthet Dent. $1991 ; 66(2): 169-76$.

14- Mehl A, Blanz V, Hickel R. A new mathematical process for the calculation of average forms of teeth. J Prosthet Dent. 2005;94(6):561-6.

15- Ogawa T, Koyano K, Suetsugu T. The relationship between inclination of the occlusal plane and jaw closing path. J Prosthet Dent. 1996;76(6):576-80.

16- Ogawa T, Koyano K, Suetsugu T. Characteristics of masticatory movement in relation to inclination of occlusal plane. J Oral Rehabil. 1997;24(9):652-7.

17- Ogawa T, Ogimoto T, Koyano K. Pattern of occlusal contacts in lateral positions: canine protection and group function validity in classifying guidance patterns. J Prosthet Dent. 1998;80(1):67-74. 18- Panek H, Matthews-Brzozowska T, Nowakowska D, Panek B, Bielicki G, Makacewicz S, et al. Dynamic occlusions in natural permanent dentition. Quintessence Int. 2008;39(4):337-42.

19- Rinchuse DJ, Kandasamy S, Sciote J. A contemporary and evidence-based view of canine protected occlusion. Am J Orthod Dentofacial Orthop. 2007;132(1):90-102.
20- Schierz O, Klinger N, Schön G, Reissmann DR. The reliability of computerized condylar path angle assessment. Int J Comput Dent. 2014;17(1):35-51.

21- Schuyler $\mathrm{CH}$. The function and importance of incisal guidance in oral rehabilitation. 1963. J Prosthet Dent. 2001;86(3):219-32. 22- Suit SR, Gibbs CH, Benz ST. Study of gliding tooth contacts during mastication. J Periodontol. 1976;47(6):331-4.

23- Thornton LJ. Anterior guidance: group function/canine guidance. A literature review. J Prosthet Dent. 1990;64(4):479-82. 24- Turp JC, Greene CS, Strub JR. Dental occlusion: a critical reflection on past, present and future concepts. J Oral Rehabil. 2008;35(6):446-53.

25- Wang M, Mehta N. A possible biomechanical role of occlusal cusp-fossa contact relationships. J Oral Rehabil. 2013;40(1):6979.

26- Weinberg LA. A cinematic study of centric and eccentric occlusions. J Prosthet Dent. 1964;14(2):290-3.

27- Woda A, Vigneron P, Kay D. Nonfunctional and functional occlusal contacts: a review of the literature. J Prosthet Dent. 1979;42(3):335-41.

28- Yaffe A, Ehrlich J. The functional range of tooth contact in lateral gliding movements. J Prosthet Dent. 1987;57(6):730-3. 